\title{
A conceptual analysis of the meaning of comfort at the end-of-life using the Walker and Avant (2014) Framework
}

\author{
Yong-Chao Hou, Fiona Timmins ${ }^{\mathrm{b}, *}$, Qian Zhoua, Ju-Zi Wanga, \\ aEmergency Department, Shanxi Provincial People’s Hospital, Taiyuan, Shanxi 030012, China \\ ${ }^{b}$ School of Nursing and Midwifery, Trinity College Dublin, Dublin 2, Ireland
}

Received: 23 July 2020; Accepted: 28 September 2020; Published: 20 June 2021

Abstract: Objective: The aim of the authors is to clarify the concept of comfort at the end-of-life in order to support understandings of fundamental nursing care needed at this stage of healthcare.

Methods: The Walker and Avant framework was applied to develop a deeper understanding of the concept of comfort at the end of life. Results: Five defining attributes of comfort in the end-of-life were identified and they are having a peaceful home-life environment, trust and consolation, proximity and social-cultural support, alleviation of suffering, and a process of integrated intervention by nurses. Conclusions: At the end-of-life patients commonly experience physical, psychological, social-cultural, and environmental discomfort. Patients' families also encounter significant challenges. However, their comfort needs are often secondary to that of the patient. Additionally, a lack of clarity exists regarding the holistic meaning of comfort at the end-of-life, which can largely be confined to understandings of physical comfort for the patient, with a limited understanding of addressing family/caregivers' needs. Therefore, this concept analysis may provide some guidance in this regard and also provides support toward a more integrated understanding of the concept.

Keywords: comfort • end-of-life care $\bullet$ concept analysis $\bullet$ death $\bullet$ dying

(c) Shanxi Medical Periodical Press.

\section{Introduction}

Despite advances in healthcare, end-of-life care is a distinct, prominent, and common stage of modern health care delivery. ${ }^{1}$ Providing comfort to patients at their end of life is one of the most important considerations for nurses during this period. ${ }^{2,3}$ While it may be difficult for patients to reach a peaceful, serene state in the final stages of life,$^{3}$ patients and families expect the nurses to deliver care intended to helping them to be comfortable and they may be distressed if this is not achieved. ${ }^{4}$ Ultimately, nurses have the responsibility and opportunity to

How to cite this article: Hou YC, Timmins F, Zhou Q, Wang JZ. A conceptual analysis of the meaning of comfort at the end-of-life using the Walker and Avant (2014) Framework. Front Nurs. 2021;2:91-98. 
enhance the comfort level of patients and their families while providing end-of-life care. ${ }^{4}$ However, while nurses may have personal understandings of what comfort means, the concept of comfort is not uniformly understood. Additionally, globally there is increasing evidence that fundamentals of care, such as comfort, receive low priority from nurses when compared with physical nursing tasks. ${ }^{5}$ Generally, these activities and supporting families are missed out completely by nurses. ${ }^{6}$ Thus, despite the importance of care at the end of life, a lack of clarity exists regarding the integrated meaning of comfort at this stage in the illness trajectory. Moreover, understandings of comfort frequently apply to physical comfort, but there is less focus on other aspects of comfort. ${ }^{7}$ At the same time treatments and invasive lifesupport frequently continue at the end of life and there may be a careful balancing act in terms of mediating the side effects of treatment and ensuring comfort. ${ }^{4,8}$ Given this complexity, and because of the lack of consistent definitions and inconsistent application to the family/ caregivers, this authors aim is to clarify the concept of comfort during end-of-life care to help inform optimal nurse interventions and how best to enhance comfort during this phase to improve quality of care.

\section{Methods}

The academic databases searched were: PubMed, CINAHL, and EMbase. The terms included in the search are "comfort," "end of life," "end life care," "terminal care," "hospice care," "hospice program," "bereavement care," and "spiritual care," and search was limited to papers in the English language only. Initially, a total of 3,932 papers ( 1,608 papers retrieved from PubMed, 952 papers retrieved from CINAHL, and 1,372 papers retrieved from EMbase) were retrieved from three databases. Then, a total of 956 duplicates were identified and removed by EndNote version X9.0. After examining the title and abstract of each paper, 2,954 papers were excluded (unrelated, or the definitions were not reported). We also utilized dictionaries to explore the concept of comfort at the end-of-life. For the final analysis, 3 online dictionaries and 22 papers were included in the concept analysis.

The Walker and Avant $^{9}$ approach of concept analysis was applied in this literature. Concept analysis is a systematic process that can clearly distinguish one concept from another that may be similar. The Walker and Avant ${ }^{9}$ framework is used widely across nursing profession internationally and provides a logical step-by-step guiding framework to support the analysis of chosen terms. The analysis within this framework involves identifying the concept, attributes, model and contrary case, antecedents, and consequences. There are eight key recommended stages $^{9}$ of the analysis outlined as follows: selecting the concept; outlining the purpose of the concept analysis; determining all possible uses of the concept; defining the attributes; identification of the model case; outlining a contrary case; identifying antecedents and consequences; and outlining the empirical referents of the defining attributes.

\section{Results}

\subsection{Use of the term}

The word comfort etymologically originates from the Latin word confortare, which means fortification, certify, corroborate, grant comfort, relieve, help, and assist. ${ }^{10}$ Moreover, the definition of comfort can include the act of comfort and providing comfort to others. ${ }^{11}$ According to the Cambridge English Dictionary, ${ }^{10}$ comfort as a noun define a person having no pain and having enough money or a feeling of ease. As a verb, comfort is defined as the act of making someone feel better when they are sad or worried. From the Bible Dictionary, ${ }^{12}$ "comfort" emphasizes encouragement, either by words or the presence of another to help in time of need. Synonymous words are to console, help, give relief, and cheer up. In the health care domain "comfort" stresses the restoration of physical, psychological, and spiritual functions within usual parameters. ${ }^{2}$

Comfort theory is also explored within a middle range nursing theory developed by Kolcaba. ${ }^{13}$ This theory integrates various concepts, including the identification of the comfort needs of patients and family members by nurses, comfort interventions, intervening variables, confounding factors, enhanced comfort, health-seeking behaviors, and institutional integrity. Further, Kolcaba ${ }^{13}$ stated that this comfort theory can be used as a general theory and can be adapted universally as well as can be further explored in terms of application to specific populations for such persons who are at the end-oflife. Therefore, it is readily applicable to the concept of comfort at the end-of-life.

Historically, comfort was described as the product of holistic nursing practice. Kolcaba and colleagues ${ }^{14}$ later constructed a theoretical framework regarding comfort to illustrate vividly how comfort theory can be applied in the health care setting to the process of care. In this framework, ${ }^{14}$ it is highlighted that nursing interventions and the comfort needs of patients and their family members should be considered. Three types of comfort are described and explained: relief, ease, and transcendence derived from four contexts: physical, psycho-spiritual, social, and environmental. This framework further outlines the comfort needs at the end-of-life to include the creation of a peaceful, 
home-life environment, trusting relationship and emotional support to patient's family, opportunity of proximity, and social-cultural support, as well as alleviating their suffering. ${ }^{14}$ Furthermore, the theory stresses that the nurse has crucial responsibility in providing comfort in the context of holistic care provision. ${ }^{14}$

There is some evidence in the literature about the measurement of patients' and family/caregivers' comfort. The Radiation Therapy Comfort Questionnaire and the Urinary Incontinence and Frequency Comfort Questionnaire, derived from the General Comfort Questionnaire (GCQ) are all well-validated methods that were identified that measure patient comfort. However, the majority of questionnaires and scoring methods were developed to measure comfort in populations with specific illnesses rather than to measure the broad concept of comfort or to address the concept broadly at end of life. For example, the Hospice Comfort Questionnaire (HCQ) is specific and is derived from the comfort theory developed by Kolcaba ${ }^{13,14}$ aimed at measuring comfort of patients and families facing end-of-life in palliative care settings. Of the 49 items in the HCQ, 18 items focus on the comfort experience of the affected family member rather than the patient directly. In addition, the HCQ was proved to be highly valid and reliable in measuring family comfort. ${ }^{15}$ Therefore, the HCQ is a useful questionnaire for measuring comfort in the end-of-life stage if it is relevant and required for those in palliative care settings.

\subsection{Determining defining attributes}

Walker and Avant $^{9}$ highlight the importance of determining defining attributes. The defining attributes of comfort at the end-of-life were identified as: having a peaceful home-life environment, trust and consolation, proximity and social-cultural support, alleviation of suffering, and a process of integrated intervention by nurses.

\subsubsection{A peaceful home-life environment}

The first attribute a peaceful home-life environment was found to be significant and essential attribute for comfort in the end-of-life stage. The results of previous study have acknowledged that an individual's satisfaction with the external environment is closely related to comfort. ${ }^{4}$ Similarly, other researchers ${ }^{16}$ have found that creating a quiet or peaceful environment is a key approach when providing physical and emotional comfort. In contrast, a loud and chaotic setting can negatively affect patients in their terminal stage. Within the hospital setting, the environment may be considered beneficially peaceful if the nurse can provide comfort for the patient for example through the use of "quiet time" procedures: dimming the lights in the patient's room and hallway; reducing unnecessary stimuli; ensuring a comfortable volume of monitoring alarms; avoiding unnecessary conversations; and ensuring that staff and visitors converse in low tones in patient care areas. ${ }^{16}$

Apart from these peaceful home-life environmental factors and in the perspective of environmental comfort, focus should be placed upon the home-life atmosphere for better comfort. Factors to be considered can range from the furniture and decoration, window views, to temperature and odor. ${ }^{2}$ Recently, a number of authors have documented that patients and their family members feel more satisfied and comfortable in a home-life type ward setting. ${ }^{2}$ Therefore, the transformation of the patient's room into an attractive, peaceful, home-like atmosphere is crucial and can be an important source of comfort, one that can be influenced by the nurse. This facilitates the promotion of comfort as it creates a space that honors both the patient and family member. Furthermore, the presence of homelike environment can positively influence patient comfort. ${ }^{7}$ Caregivers can consequently promote peace during the final hours or days of a patient's life in such an environment. ${ }^{17}$ Although providing a major homelike environment might have financial implications in a hospital setting, and it is only feasible within a major project, where a nurse can do a lot to create this environment by, for example, encouraging the use of personal items.

\subsubsection{Trust and consolation}

Trust and consolation were the second defining attribute for comfort at the end-of-life. One research study confirmed that having a trusting relationship between a nurse and the patient and their family can share and relieve the burden of suffering. ${ }^{18}$ In addition, this study suggested that consolation can only occur in an environment of openness, presence, and availability. Thus, nurses can deliver a high level of comfort, care, and consolation when a close and trusting relationship is developed with the patient and family/ caregiver. ${ }^{19}$ For example, physical contact is an effective intervention which can help transcend any existing barriers, establish trust and promote comfort regardless of the person's state of health. ${ }^{2}$ Similarly, according to research concerned to the care for a dying child, nurses who had been able to build a relationship and trust with the family were able to provide consolation and a comforting environment for the family and patient. ${ }^{18}$

\subsubsection{Comfort at the end-of-life}

The third defining attribute of comfort in the end-of-life stage is proximity and social-cultural support. This is a phenomenon that has changed relatively recently. 
Previously, the majority of people died at home where they were surrounded and cared for by loved ones. ${ }^{20}$ However, the circumstances of dying have changed profoundly to a more unfamiliar hospital setting where patients are not close to their families. This has resulted in an increase in discomfort for patients. ${ }^{21}$ It is important to note the finding that social-cultural comfort needs pertain to interpersonal, family, and societal relationships, including family traditions, culture, rituals, religion, and faith. ${ }^{14}$ Social-cultural comforts are adversely influenced by lack of respect for privacy and a limited level of social support and relationships with family. ${ }^{22}$ Optimal care and comfort is thus achieved by allowing the dying patient to be surrounded by their loved ones in the final hours of their death. ${ }^{3}$ Only by attaining the proximity to their family member can the nurse fully support them in this social-cultural aspect of providing comfort.

\subsubsection{Alleviating the suffering of both patients and their family}

The fourth defining attribute is alleviating the suffering of both patients and their family members. The end-oflife situation puts lot of pressures on patients and family members such as the presence of a life-threatening illness, changes in responsibilities, changes in social life, and uncertainty when faced with the unknown factors. ${ }^{23}$ Their suffering can therefore be a multidimensional experience of pain, sorrow, fear, and vulnerability associated with physiological and psychological difficulties. ${ }^{18}$ To relieve or reverse physical discomforts for patents, thereby improving their state of comfort, will promote dying with tranquility. ${ }^{3}$ Physical care including minimization of pain and discomfort with medication or lifesupport techniques are thus become important sources of comfort that allow patients to die with greater dignity. However, patients and their families are both encountered by psychological discomfort, derived from emotional distress. ${ }^{24}$ Bereavement presents considerable challenges around experiences of loss and life transition, particularly for family members. ${ }^{23}$ Consequently, the nurse must react to the demands of end-of-life patients and their family members for both physical and psychological care with appropriate interventions to alleviate suffering and promote comfort.

A process of integrated intervention by the nurse is the final defining attribute for comfort in the end-of-life stage. It is widely accepted that nurses have a significant role in end-of-life care.$^{25}$ The role of the nurse is to identify the needs of patients and their families at this stage and has to plan, implement, and evaluate appropriate interventions. ${ }^{26}$ Hence, comfort at the end-of-life stage is not a static provision but rather it is a dynamic process of integrated intervention by nurses to influence the surrounding environment, patients, and families, with continuous evaluation of the effectiveness of interventions.

\subsection{Operational definitions}

As a result of the combination of these attributes, the operational definition of comfort at the end-of-life stage was developed. Comfort in the end-of-life stage is defined as an integrated process of nursing interventions, in which nurses create a peaceful, home-life environment, build trust and deliver emotional support to the patient's family/caregivers, which allow for proximity and socialcultural support between the patient and family, as well as alleviate their physical suffering.

\subsection{Exemplary cases}

Walker and Avant $^{9}$ highlight the need within the concept analysis to identify both a model and a contrary case to indicate the precise use of the concept in practice and promote discrete understanding. In the following case, an unconsciousness patient is selected to emphasize the interactional engagement of patients, patients' family members, and nurses synchronously in end-of-life care rather than focusing solely on the patients' needs.

\subsubsection{Model case}

Mr. Li suffered from terminal lung cancer. One day, when his condition worsened, the nurse contacted his family to say that his condition had deteriorated. When Mrs. Li arrived to the unit, she felt so sad that she could not help weeping and the nurse gave her a reassuring hand and comforted her (evoking trust and providing consolation). Shortly afterward the family made a decision based on the advanced care directives to cease advanced life support measures, and active treatments other than those for palliative purposes. Having taken some time out, they re-entered Mr. Li's room to find the atmosphere in the room changed completely. There was a small table placed near the bed with a soft-light lamp. Several lightly scented candles were placed around, and the room appeared very peaceful. The bed was covered with a colorful quilt, and a fresh, clean pillow was placed under his head (to promote a peaceful, home-life environment). Mr. Li was dressed in clean clothes, and he was in a state of peacefulness and comfortable position, and all nonessential equipment was removed (to alleviating suffering). Mr. Li was provided with oxygen and observed for signs of pain and appropriate analgesia was provided (to alleviating suffering). The nurse left the family alone with the patient for about 30 minutes. Mrs. Li put a bible beside his head and prayed. After that, Mrs. 
Li was deeply grateful for everything that the nurse has done (providing the opportunity of proximity and socialcultural support). In this process, the nurse, as a caregiver, delivered quality fundamental holistic care across many levels (a process of integrated intervention).

\subsubsection{Contrary case}

Mr. Gurkova experienced a road traffic accident and arrived at the emergency department (ED). The large ED has stretchers that were arranged side by side with only a curtain between each stretcher providing minimal or no privacy (no provision of a peaceful, home-life environment). His condition deteriorated and the nurse immediately informed his son in a cold manner: "his condition is worsening; we can't let you in yet, are you his next of kin" (no provision of trust and consolation). His son felt frustrated, sad, and hopelessly sitting in the waiting area of the ED (no provision for opportunity of proximity and social-cultural support).Then after some time, the son was informed that Mr. Gurkova's condition had deteriorated significantly and he was unlikely to survive. When Mr. Gurkova's son was finally permitted to see him, Mr. Gurkova had died, his bloodstained clothes lay on the stretcher in disorder, and the room was in disarray (no provision for alleviating their suffering). Moreover, it is observed that the nurse did not perform her comfort role at end-of-life with due care (no provision for a process of integrated intervention).

\section{Discussion}

The end-of-life elicits suffering. ${ }^{27-29}$ However the concept analysis clearly demonstrates that engaging family members in the process of end-of-life care facilitates strengthens the effects of the comforting interventions by the nurse. ${ }^{11}$ Furthermore, patients often have complex comfort needs at the end-of-life stage in addition to the need for careful physical symptom management. ${ }^{7}$ There is an expectation from patients and families that the nurses should act as caregivers to fulfill requests for comfort. ${ }^{4}$ Frustrations can arise if this is not the case, as families perceive inadequacies in care and may complain. ${ }^{30}$ However, when nurses transform the patient's room into a peaceful and home-like atmosphere for example, this can make a tremendous difference to the feeling of comfort, improve the families' psychosocial wellbeing, though these matters are not costly. ${ }^{17}$ Of course, supportive interventions by the nurse are more effective when the nurse is experienced and skilled in technical competence and human competence. ${ }^{3}$ In other words, confidence in the technical quality of care can inspire assurance that the process of end-of-life care will be operated with the least amount of suffering for the patient. At the same time, knowing precisely what to do may not necessarily translate into enacting these comfort measures, perhaps due to environmental or personal reasons. Nurses are encouraged in these circumstances to reflect on their personal practice and consider ways so that it could be improved. ${ }^{30,31}$ It is possible for example to determine what more could be done in personal practice to display comfort, or build trust, and having the capacity to reflect and learn from this reflection is crucial. ${ }^{31}$

The World Health Organization ${ }^{32}$ recommends that patients at the end-of-life stage should have a high quality of life mediated by comfort care including comprehensive intervention for pain and other physical and psychosocial needs. The philosophy of providing comfort is an approach that seeks to improve the feeling of peace in dying people and their families. ${ }^{3}$ The general consensus in the literature is that promotion of comfort in the end-of-life stage results in a peaceful death, which can be accomplished by surrounding patients with their kith and kin, and thus ensuring their dignity. $3,7,16,17$ Consequently, providing comfort is the best way for nurses to show compassion and humanity at the end-of-life stage, as well as increasing the satisfaction of patients and their families. ${ }^{17}$

Understandings of comfort also require initiatives to promote assessment of comfort needs. While detailed measurement instruments such as the $\mathrm{HCQ},{ }^{13,14,33}$ are available to measure the concept of comfort at the endof-life stage, the use of these tools to assess patient or family comfort, is overly burdensome at this point given their level of detail and clear research purpose. More straightforward screening tools to assess patients and family/caregiver needs would likely be useful in this regard. For example staff at Wuhan Union Hospital, in the People's Republic of China ${ }^{34}$ recently highlighted such an approach, suggesting that the nurse spend time asking key questions such as "Do you have any discomfort?" and "Do you have any needs that I need to address?" Similarly, Ross and McSherry ${ }^{35}$ recommend a two-step approach to assessment (directly in relation to spiritual care but also relevant here), which are "What is important to you right now?' and 'How can we help?" These general, simple questions might form the basis of a useful assessment of patient and family/caregiver needs in relation to comfort requirements at the end of life.

\section{Conclusions}

The end-of-life can be distressing for both patients and their family/caregivers. Therefore, the pursuit of comfort is a substantive need and primary goal in end-of-life care of patients and their families. The recent COVID 19 global pandemic extenuates many of the barriers to 
comfort outlined in this concept analysis. Visitor restrictions, lack of physical contact, restrictions on personal items, all contravene comfort requirements and are likely to worsen the distress during this time. It is vitally important that the conceptual clarity provided in this paper raises awareness about the patient and family comfort needs at end of life, and aim to support these needs in creative ways. The five defining attributes of comfort in the end-of-life were identified as: having a peaceful home-life environment; trust and consolation; proximity and social-cultural support; alleviation of suffering; and a process of integrated intervention by nurses. A more holistic meaning of comfort at the end-of-life, is needed that goes beyond providing physical comfort for the patient, with an extended consideration

\section{References}

1. Mitchell S, Dale J. Advance care planning in palliative care: a qualitative investigation into the perspective of paediatric intensive care unit staff. Palliative Med. 2015;29:371-379.

2. deAzevedo Ponte KM, Da Silva LDF. Comfort as a result of nursing care: an integrative review. Revista de Pesquisa: Cuidado é Fundamental Online. 2015;7:2603.

3. Souza da Silva R, Pereira Á, Carneiro Mussi F. Comfort for a good death: perspective nursing staff's of intensive care. Escola Anna Nery Revista de Enfermagem. 2015;19:40-46.

4. Tuti N, Gayatri D, Rachmawati M. Comfort assessment of cancer patient in palliative care: a nursing perspective. Int J Caring Sci. 2017;10:209.

5. Feo R, Kitson A, Conroy T. How fundamental aspects of nursing care are defined in the literature: a scoping review. J Clin Nurs. 2018;27:2189-2229.

6. Sasso L, Bagnasco A, Zanini M, et al. The general results of the RN4CAST survey in Italy. J Adv Nurs. 2017;73:2028-2030.

7. Coelho A, Parola V, Escobar-Bravo M, Apóstolo J. Comfort experience in palliative care: a phenomenological study. BMC Palliative Care. 2016;15:71.

8. Ai ZP, Gao XL, Li JF, Zhou JR, Wu YF. Changing trends and influencing factors of the quality of life of chemotherapy patients with breast cancer. Front Nurs. 2017;4:18-23.

9. Walker LO, Avant KC. Strategies for Theory Construction in Nursing. 5th ed. London: Pearson New International Edition; 2014.

10. Cambridge English Dictionary. https://dictionary.cambridge.org/dictionary/english/comfort. Accessed March 6, 2020. of family/caregivers' needs. While an integrated approach to comfort is challenging at this time, nurses still need to be cognizant of the needs to ensure that comfort provision requires physical, psychological, and social care, as well as should aim to provide such care in straightforward, safe ways with things that are available to them.

\section{Ethical approval}

Ethical issues are not involved in this paper.

\section{Conflicts of interest}

All contributing authors declare no conflicts of interest.
11. da Durante ALTC, Tonini T, Armini LR. Comfort in palliative care: the know-how of nurses in general hospital. J Nurs UFPE on line. 2014;8:530-536.

12. Bible Dictionary. http://www.biblestudytools.com/ dictionary/comfort/. Accessed March 6, 2020.

13. Kolcaba K. Comfort Theory and Practice. A Vision for Holistic Health Care and Research. New York: Springer; 2003.

14. Kolcaba K, Tilton C, Drouin C. Comfort theory: a unifying framework to enhance the practice environment. J Nurs Adm. 2006;36:538-544.

15. Kolcaba KYA. Taxonomic structure for the concept comfort. Image J Nurs Scholarsh. 1991;23:237-240.

16. Krinsky R, Murillo I, Johnson J. A practical application of Katharine Kolcaba's comfort theory to cardiac patients. Appl Nurs Res. 2014;27:147-150.

17. Dreher BS. All the comforts of home: transformation to a comfort environment in critical care. Crit Care Nurse. 2017;37:78-80.

18. Ångström-Brännström C, Dahlqvist V, Strandberg $G$, Norberg A. Descriptions of comfort in the social networks surrounding a dying child. Nordic J Nurs Res. 2014;34:4-8.

19. Rijnaard MD, van Hoof J, Janssen BM, et al. The factors influencing the sense of home in nursing homes: a systematic review from the perspective of residents. J Aging Res. 2016: 2016:6143645.

20. Cook D, Rocker G. Dying with dignity in the intensive care unit. N Engl J Med. 2014;370:2506-2514.

21. Sepúlveda Sánchez JM, Canca Sánchez JC, Pérez Trueba E, Rueda Dominguez A, Morales Asencio JM, Morales Gil IM. Impact of a legislative framework on quality of end-of-life care and dying 
in an acute hospital in Spain. Int $J$ Palliat Nurs. 2014;20:225-231.

22. Slatyer S, Pienaar C, Williams AM, Proctor K, Hewitt L. Finding privacy from a public death: a qualitative exploration of how a dedicated space for endof-life care in an acute hospital impacts on dying patients and their families. J Clin Nurs. 2015;24: 2164-2174.

23. Lundberg T, Olsson M, Fürst CJ. The perspectives of bereaved family members on their experiences of support in palliative care. Int $J$ Palliat Nurs. 2013;19:282-288.

24. Ramanayake RP, Dilanka GV, Premasiri LW. Palliative care; role of family physicians. J Family Med Prim Care. 2016;5:234-237.

25. Corcoran K. Evaluation of an educational workshop to increase comfort levels of professional caregivers with end-of-life care. Medsurg Nurs. 2016;25:103-109.

26. Cameron D, Johnston B. Development of a questionnaire to measure the key attributes of the community palliative care specialist nurse role. Int J Palliat Nurs. 2015;21:87-95.

27. Ringdal GI, Jordhøy MS, Kaasa S. Family satisfaction with end-of-life care for cancer patients in a cluster randomized trial. J Pain Symptom Manag. 2002;24:53-63.

28. Freitas KS, Menezes IG, Mussi FC. Comfort from the perspective of families of people hospitalized in the Intensive Care Unit. Texto \& Contexto-Enfermagem. 2012;21:896-904.

29. Kisvetrová H, Vévodová Š, Školoudík D. Comfortsupporting nursing activities for end-of-life patients in an institutionalized environment. J Nurs Scholarsh. 2017;50:126-133.

30. Lyu CM, Zhang L. Critical emancipatory reflection on a practice-based issue in relation to nurses' communicative role with unsatisfied clients in Chinese hospitals. Front Nurs. 2019;6:41-46.

31. Lai YL. Critical emancipatory reflection on establishing an equal, trusting relationship among surgery participants in clinical practice in China. Front Nurs. 2019;6:47-52.

32. World Health Organization. Palliative care; 2014 http://www.who.int/cancer/palliative/en/. Accessed March 3, 2020.

33. Novak B, Kolcaba K, Steiner R, Dowd T. Measuring comfort in caregivers and patients during late end of life care. Am J Palliat Care. 2001;18:170-180.

34. Liu $Y$, Wu $X$, Timmins F. Strengthening actions for caring as a core component of nursing in the People's Republic of China. J Nurs Manag. 2019;27:1577-1579.

35. Ross L, McSherry W. Two questions that ensure person-centred spiritual care. Nursing Standard; 2018. https://rcni.com/nursing-standard/features/ two-questions-ensure-person-centred-spiritualcare-137261. Accessed March 3, 2020. 